\section{Cardio-Pulmonale Reanimation und Früh-Defibrillation}

\author{
Einführung der neuen Richtlinien \\ durch den Swiss Resuscitation Council
}

M. von Planta, J. J. Osterwalder

\begin{abstract}
Einleitung
1960 wurde ein alter Menschheitstraum wahr. Die Wissenschaft hatte endlich einen Weg gefunden, den plötzlichen Herztod aufzuhalten. Das Erstaunliche daran ist, dass ein Paar Hände, eine funktionierende Lunge, eine durchschnittliche Begabung und etwas Übung einen wesentlichen Beitrag an dieses Wunder leisten kann. Mit der Entwicklung automatischer externer Defibrillatoren (AED) ist ein weiterer Meilenstein im Kampf gegen den Kreislaufstillstand dazugekommen. Diese AED sind auch von wenig trainierten Laien einsetzbar. Sie ermöglichen als alleinig wirksame Behandlungsmöglichkeit die häufigste Ursache des unerwarteten Kreislaufstillstands - das Kammerflimmern (KF) - effizient zu beenden.

Die Rate von überlebenden Menschen ohne schwere Folgeschäden ist noch sehr klein (z.B. New York und Chicago 1-2\%, ausgewählte Spitäler der Schweiz der Jahre 1992-1996: 13\%, Seattle und Minnesota 25-46\%). Die guten Resultate aus Seattle und Minnesota sind Folgen der an diesen Orten lückenlos funktionierenden Überlebenskette [1, 2].
\end{abstract}

\section{Abbildung 1}

Chain of Survival (Überlebenskette).

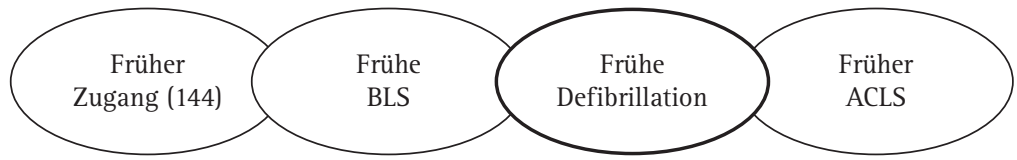

Korrespondenz:

Prof. Dr. med. Martin von Planta

St. Johanns-Vorstadt 44

CH-4056 Basel

Dr. med. Joseph J. Osterwalder

Kantonsspital St. Gallen

Rorschacherstr. 95

CH-9007 St. Gallen
Die raschen Handlungen der Überlebenskette: Alarm über Notrufnummer 144, Basic Life Support (BLS), Defibrillation, Advanced Cardiovascular Life Support (ACLS) und Transport ins Spital sowie Intensivpflege im Spital gelten seit Anfang 90er Jahre auch für die Schweiz.

40 Jahre nach Einführung der Cardio-Pulmonalen Reanimation (CPR) und mit der revolutionären Entwicklung von AED ist es Zeit, dass die Schweiz aus der Reanimationslethargie erwacht und die angebotenen Chancen wahrnimmt. Unser Ziel: Jeder Bürger soll in BLS-AED (Basic life support - automatische externe Defibrillation) ausgebildet werden. Nur so kann das Überleben von Patienten im Falle eines Kreislaufstillstands ausserhalb des Spitals von weniger als 5\% auf 20-30\% ansteigen. Solch günstige Überlebensraten werden erreicht, wenn die Überlebenskette lückenlos funktioniert (Abb. 1).

Die hier vorgelegten Richtlinien entsprechen den gültigen internationalen Empfehlungen der American Heart Association (AHA) in Zusammenarbeit mit dem International Liaison Committee on Resuscitation (ILCOR), wo auch der European Resuscitation Council (ERC) Mitglied ist. Es ist zu hoffen, dass ihre Anwendung den vom Kreislaufstillstand betroffenen Menschen zugute kommt. Dieser Text stellt die neuen schweizerischen BLS-AED-Richtlinien für Kinder ab 8 Jahren sowie für Erwachsene vor. Er basiert auf den internationalen Richtlinien 2000 des ERC und der AHA [3, 4].

\section{Epidemiologie und Ursachen}

Solide statistische Kenndaten für die Schweiz fehlen weitgehend. Die nachfolgenden Aussagen sind deshalb Schätzungen auf der Grundlage ausländischer Untersuchungen:

- Alle 1-2 Stunden tritt in der Schweiz ein Kreislaufstillstand ausserhalb des Spitals auf.

- Etwa 80\% der erfolgreich wiederbelebten Patienten werden im KF angetroffen.

- Rund 70\% der Kreislaufstillstände treten im häuslichen Umfeld auf.

- Exakte Überlebensraten von Personen mit Kreislaufstillstand sind derzeit gesamtschweizerisch nicht bekannt. Sie liegen wahrscheinlich unter 5\% und im günstigsten Fall höchstens bei 13\%.

- Pro Minute unbehandeltem KF sinkt die Überlebenschance durchschnittlich um 7-10\%.

- Ersthelfer-BLS verbessert die Überlebenschance um das 2-3fache, zusammen mit AED sogar um das 3-10fache.

- Nach durchschnittlich 8-12 Minuten unbehandeltem KF kommt es zum feinen KF mit kleiner Amplitude und schliesslich zur Asystolie (Nulllinie) mit sehr schlechter Prognose (Abb. 2). 


\section{Abbildung 2}

Übergang von unbehandeltem Kammerflimmern in Asystolie.

Nach 8-12 Minuten unbehandeltem KF kommt es zum feinen KF mit kleiner Amplitude und schliesslich zur Asystolie (Nullinie).

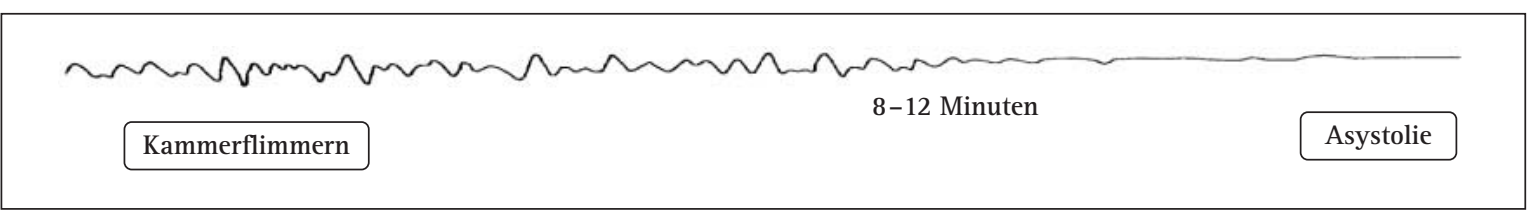

\section{Neue CPR-Richtlinien}

Die Richtlinien zur CPR wurden erstmals international einheitlich gestaltet und synchron in Europa [3] und den USA [4] publiziert. Somit gibt es zwischen Europa (ERC) und den USA (AHA) keine Unterschiede mehr, was die Umsetzung in praktische Kurse sehr vereinfacht.

Der wesentliche Fortschritt dabei ist die Abstützung der Richtlinien auf die Pathophysiologie kardiovaskulärer Ereignisse. Dabei wurde der zerebrovaskuläre Insult in der Priorität von Erkennung, Erstbehandlung und definitiver Therapie dem akuten koronaren Syndrom mit seiner gravierendsten Ausprägung - dem Kreislaufstillstand - gleichgestellt. Neue Entwicklungen in der Behandlung von zerebrovaskulären Insulten und Herzstillständen wurden in einem aufwendigen Verfahren Evidenz-basiert evaluiert.

Die entscheidenden Unterschiede zu den Richtlinien von 1992 [5, 6] sind in Tabelle 1 aufgeführt. Deren Begründung ist in den 2000 Richtlinien ausführlich abgehandelt [3, 4]. Diese Richtlinien werden auch über Internet zugänglich sein (www.erc.edu oder www.americanheart.org).

\section{Swiss Resuscitation Council (SRC)}

Auf Initiative der Swiss Medical Rescue and Emergency Conference (SMEDREC) wurde am 16. Juni 2000 anlässlich der Jahrestagung der Schweizerischen Gesellschaft für Notfall- und Rettungsmedizin (SGNOR) in La Chaux-de-Fonds der SRC gegründet. Der SRC ist eine interdisziplinäre Plattform in der Rechtsform eines unabhängigen Vereins, welcher sich ausschliesslich mit der CPR befasst.

\section{Zielsetzungen des SRC}

- Die Verbesserung der Qualität von prähospitaler und intrahospitaler CPR;

- das Konzept der Rettungskette zu verbreiten;

- die Instruktion in CPR zu koordinieren und eine unité de doctrine zu schaffen;

- im Kontakt mit ausländischen Organisationen Evidenz-basierte Richtlinien zu erarbeiten.

\section{Vorstand}

Ein erster Vorstand begann mit den Geschäften. Der Vorstand soll entsprechend der Entwicklung des SRC weiter wachsen. Folgende Vorstandsmitglieder leiten derzeit den SRC:

\begin{tabular}{|lll|}
\hline Name & Funktion & Verbindung zu \\
\hline Prof. Dr. Martin von Planta, Basel & Präsident & ERC, SGIM \\
\hline Dr. Joseph Osterwalder, St. Gallen & Vizepräsident & AHA, SMEDREC \\
\hline Daniel Thüring, Walenstadt & Kassier & VRS \\
\hline Dr. Martin Brüesch, Zürich & Beisitzer & SGAR \\
\hline Dr. P. Eckert, Sion & Beisitzer & SGI \\
\hline Dr. Nils Gueissaz, Romont & Beisitzer & SGAM \\
\hline Dr. Daniel Hodel, Luzern & Beisitzer & SGNOR \\
\hline Dr. Erwin Oechslin, Zürich & Beisitzer & SGK \\
\hline Dr. Philippe Reigner, Lausanne & Beisitzer & SRK \\
\hline Dr. G. Savary, Russo & Beisitzer & REGA \\
\hline Alfons Scherrer, Zürich & Beisitzer & SBK \\
\hline Jessica Soldati, Bern & Sekretariat & FMH \\
\hline
\end{tabular}




\section{EDV}

Eine Webseite "www.resuscitation.ch" und eine E-mail-Adresse «info@resuscitation.ch»wurden geschaffen. Die Richtlinien des SRC werden darauf erscheinen und den Benutzern gratis zu Verfügung gestellt. Es werden auch Links auf die Seiten anderer im Reanimationsbereich aktiver Organisationen zur Verfügung stehen, um deren Richtlinien greifbar zu machen.

\section{BLS-AED-Algorithmus und Vorgehen (Abb. 3 und 4)}

A bedeutet Atemwege, B Beatmung, C Cirkulation/ Compression und D Defibrillation. Das Leitsystem wird klinisch überprüft, was je nach Ergebnis in der spezifischen Behandlung mündet. Somit wird das in der Schweiz weit verbreitete "GABI" durch das ABCD abgelöst (Abb. 6).

Für das primäre $A B C D$ von Kindern über 8 Jahren und Erwachsenen gelten 2 Grundsätze:

- Die Reihenfolge des ABCD ist verbindlich, d.h., A hat Priorität vor B, B vor C.

- Sobald ein lebensbedrohlicher Befund auf einer Stufe festgestellt wird, folgt eine klar definierte sten Stufe übergeht. So wird z.B. zuerst das Erbrochene im Mund ausgewischt, bevor man B überprüfen kann.

Im Zentrum stehen BLS und Defibrillation

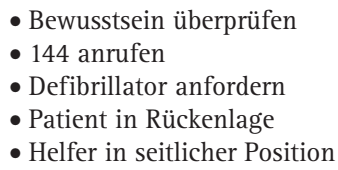
lebensrettende Massnahme, bevor man zur näch-

- Rasche Defibrillation durch Laien mit AED für Kinder ab 8 Jahren sowie Erwachsene.

- Unterricht von Kindern ab 10-12 Jahren in BLS hat hohe Priorität.

\section{Umsetzung der Richtlinien}

\section{BLS-AED-Richtlinien}

Der SRC hat im vergangenen Jahr die Anpassung an die neuen Richtlinien vorgenommen und stellt sie nachfolgend mit dem begleitenden Merkblatt erstmals vor.

\section{Laien-Frühdefibrillation}

(Public Access Defibrillation)

Die bestehenden Richtlinien von 1995 wurden überarbeitet. Sie wurden im Frühsommer 2001 publiziert und sind auch über Internet abrufbar [10].

\section{Umsetzung AHA/ERC-Richtlinien für ACLS/PALS}

Das 2000 "Handbook of Emergency Cardiovascular Care" der AHA wird von der SGNOR übersetzt und voraussichtlich Anfang 2002 verfügbar sein. Die Instruktoren von ACLS- und PALS-Kursen werden bis Ende 2001 auf die neuen Richtlinien umgeschult sein.

\section{Projekt EXP0.02}

An der EXP0.02 soll ein Reanimationskurs nach den neuen CPR-Richtlinien angeboten werden, welcher auch von der Schweizerischen Herzstiftung, der Armee und anderen Organisationen mitgetragen wird.

\section{Kurswesen}

Der SRC hat Richtlinien für Laien-AED-Kurse und professionelle Halbautomaten-Kurse herausgegeben. Kurszentren, welche diese Richtlinien einhalten, können nach entsprechender Überprüfung durch den SRC das Logo des SRC für die Zertifikate verwenden. Die Richtlinien sind im Internet unter www.resuscitation. ch abrufbar.

Wichtigste Änderungen seit 1992 (Tab. 1)

- Die Beatmung kann unter schwierigen Umständen (unbekannter Patient, Infektionsgefahr, wenig Training) in den ersten 6-12 Minuten einer CPR weggelassen werden. Thoraxkompression (früher Herzmassage) alleine ist nämlich besser als keine CPR. Da aber rund 70\% der Kreislaufstillstände im häuslichen Umfeld auftreten, sind für solche Situationen die obigen Bedenken weniger relevant.

- Bei Atemwegsverlegungen durch einen Fremdkörper mit Bewusstlosigkeit genügt die Thoraxkompression für Laien. Professionelle Helfer werden weiterhin im Heimlich-Manöver ausgebildet.

- Beatmung durchführen, bis sich der Brustkorb deutlich hebt (etwa 700-1000 ml Atemzugvolumen).

- Keine Pulskontrolle für Laien (ausser bei AED-Anwendung).

- Das Verhältnis von Thoraxkompression zu Beatmung beträgt neu 15:2, unabhängig ob in der 1- oder 2-Helfer-Methode.

\section{Legale Aspekte}

- Erstretter, welche mit einer Reanimation beginnen, reanimieren bis:

- der sichere Tod festgestellt ist oder eine klare Patientenverfügung vorliegt;

- ein genügender Spontanpuls sowie eine genügende Atemlage erreicht wurden;

- ein professionelles Rettungsteam die Reanimation übernimmt;

- ein Arzt die Reanimation übernimmt und sich zum Abbruch entschliesst;

- der Ersthelfer erschöpft ist oder gefährliche Umwelteinflüsse für den Ersthelfer vorliegen.

- Bisher wurde noch nie ein Laienhelfer wegen BLSMisserfolg gerichtlich belangt.

- AED kann als delegiertes Verfahren auch von medizinischen Laien unter ärztlicher Kontrolle durchgeführt werden. 


\section{Tabelle 1}

Wichtigste Änderungen der CPR-Richtlinien.

Generell gilt: Zerebrovaskuläre Insulte haben gleiche Priorität wie akute koronare Syndrome.

\begin{tabular}{|l|l|}
\hline $\begin{array}{l}\text { BASIC LIFE SUPPORT (BLS) } \\
\text { (Abb. 3 und 4) }\end{array}$ & $\begin{array}{l}\text { ADVANCED CARDIOVASCULAR LIFE SUPPORT (ACLS) } \\
\text { (Abb. 5) }\end{array}$ \\
\hline \multicolumn{2}{|c|}{ Beatmung kann in den ersten 6-12 Minuten der CPR wegfallen, wenn die Umstände schwierig sind. } \\
Thoraxkompression alleine ist besser als keine CPR [7].
\end{tabular}

\section{Schlussfolgerungen}

Es gibt nur eine Pathophysiologie. Darum sind die ERC- und AHA-Richtlinien einheitlich. Deren Anwendung kann an lokale Bedürfnisse angepasst werden. Die gestaffelte Einführung in der Schweiz ist im zeitlichen Einklang mit derjenigen des ERC und der AHA. Somit ist eine unnötige Hast nicht angebracht! Die Umstellung soll in der Schweiz gemäss Tabelle 2 auf den 1.1.2002 erfolgen und bis zum 31.12.2002 abgeschlossen sein. Das ehemalige GABI wird dabei durch das ABCD abgelöst (Abb. 6).

\section{Tabelle 2}

SRC Aktionsplan.

\begin{tabular}{|c|c|c|c|}
\hline Dokumente & Datum & Web & Verantwortung \\
\hline BLS-AED-Merkblatt & Mai 01 & \multirow{5}{*}{$\begin{array}{l}\text { Dokumente } \\
\text { als PDF files } \\
\text { auf SRC Website }\end{array}$} & SRC \\
\hline BLS-AED-Richtlinien & Juni 01 & & SRC \\
\hline Defibrillations-Broschüre & Oktober 01 & & SRC, SMEDREC \\
\hline ACLS-Richtlinien & Anfang 02 & & ACLS-Arbeitsgruppe \\
\hline PALS-Richtlinien & Anfang 02 & & PALS-Arbeitsgruppe \\
\hline
\end{tabular}

\section{Literatur}

1 White RD, Hankins DG, Atkinson EJ. Patient outcomes following defibrillation with low energy biphasic truncated exponential waveform in out-of-hospital cardiac arrest. Resuscitation 2001;49:9-14.

2 Eisenberg MS, Mengert TJ. Cardiac Resuscitation. N Engl J Med 2001;344:1304-13.

3 Guidelines 2000 for Cardiopulmonary Resuscitation and Emergency Cardiovascular Care. An International Consensus on Science. Resuscitation 2000;46:1-448.

4 American Heart Association in Collaboration with the International Liaison Committee on Resuscitation. Guidelines 2000 for Cardiopulmonary Resuscitation and Emergency Cardiovascular Care. Circulation 2000;102 (Suppl I):1-384.

5 American Heart Association. Guidelines for Cardiopulmonary Resuscitation and Emergency Cardiac Care. JAMA 1992; 268:2171-303.

6 European Resuscitation Council. Guidelines for Resuscitation, 64 pg. Antwerp, 1994.

7 Hallstrom A, Cobb L, Johnson E, Copass M. Cardiopulmonary resuscitation by chest compression alone or with mouth-tomouth ventilation. N Engl J Med 2000;342:1546-13.

8 Lindner KH, Dirks B, Strohmenger HU, et al. Randomised comparison of epinephrine and vasopressin in patients with out-of-hospital ventricular fibrillation. Lancet 1997;349: 535-7.

9 Kudenchuck PJ, Cobb LA, Copass MK, et al. Amiodarone for resuscitation after out-of-hospital cardiac arrest due to ventricular fibrillation. N Engl J Med 1999;341:871-8.

10 Swiss Resuscitation Council, 2001. Richtlinien und Empfehlungen zur Ausbildung und Anwendung der Defibrillation mit automatischen und halbautomatischen externen Defibrillatoren (AED). (www.resuscitation.ch). 


\section{Glossar der Abkürzungen}

\begin{tabular}{|c|c|}
\hline Abkürzung & Bedeutung \\
\hline $\mathrm{ABCD}$ & $\begin{array}{l}\text { Das primäre ABCD: } \\
\text { Airway (Atemwege) - Breathing (Beatmung) - Circulation - Defibrillation }\end{array}$ \\
\hline ACLS & Advanced Cardiovascular Life Support \\
\hline AED & $\begin{array}{l}\text { Automated External Defibrillator (automatischer externer Defibrillator), } \\
\text { Automatische externe Defibrillation }\end{array}$ \\
\hline AHA & American Heart Association \\
\hline AICD & $\begin{array}{l}\text { Automated Internal Cardioverter/Defibrillator: } \\
\text { Automatischer interner Kardioverter/Defibrillator }\end{array}$ \\
\hline BLS & $\begin{array}{l}\text { Basic Life Support: Beatmung und Thoraxkompression } \\
\text { (CPR = Cardiopulmonary Resuscitation wird häufig als Synonym verwendet) }\end{array}$ \\
\hline ERC & European Resuscitation Council \\
\hline FDA & Federal Drug Administration \\
\hline FMH & Foederatio Medicorum Helveticorum \\
\hline ILCOR & International Liaison Committee on Resuscitation \\
\hline IVR & Interverband für Rettungswesen \\
\hline KF & Kammerflimmern \\
\hline KT & Kammertachykardie \\
\hline KVG & Krankenversicherungsgesetz \\
\hline PALS & Pediatric Advanced Life Support \\
\hline REGA & Schweizerische Rettungsflugwacht \\
\hline SBK & Schweizerischer Berufsverband für Krankenpflege \\
\hline SGNOR & Schweizerische Gesellschaft für Notfall- und Rettungsmedizin \\
\hline SGAM & Schweizerische Gesellschaft für Allgemeine Medizin \\
\hline SGAR & Schweizerische Gesellschaft für Anästhesie und Reanimation \\
\hline SGI & Schweizerische Gesellschaft für Intensivmedizin \\
\hline SGIM & Schweizerische Gesellschaft für Innere Medizin \\
\hline SGK & Schweizerische Gesellschaft für Kardiologie \\
\hline SMEDREC & $\begin{array}{l}\text { Swiss Medical Rescue and Emergency Conference } \\
\text { Schweizerische Medizinische Rettungskommission }\end{array}$ \\
\hline SRC & Swiss Resuscitation Council \\
\hline SRK & Schweizerisches Rotes Kreuz \\
\hline VRS & Verein Rettungssanitäter Schweiz \\
\hline
\end{tabular}




\section{Abbildung 3}

BLS-AED Algorithmus bei Kreislaufstillstand. Für Kinder älter als 8 Jahre und Erwachsene.
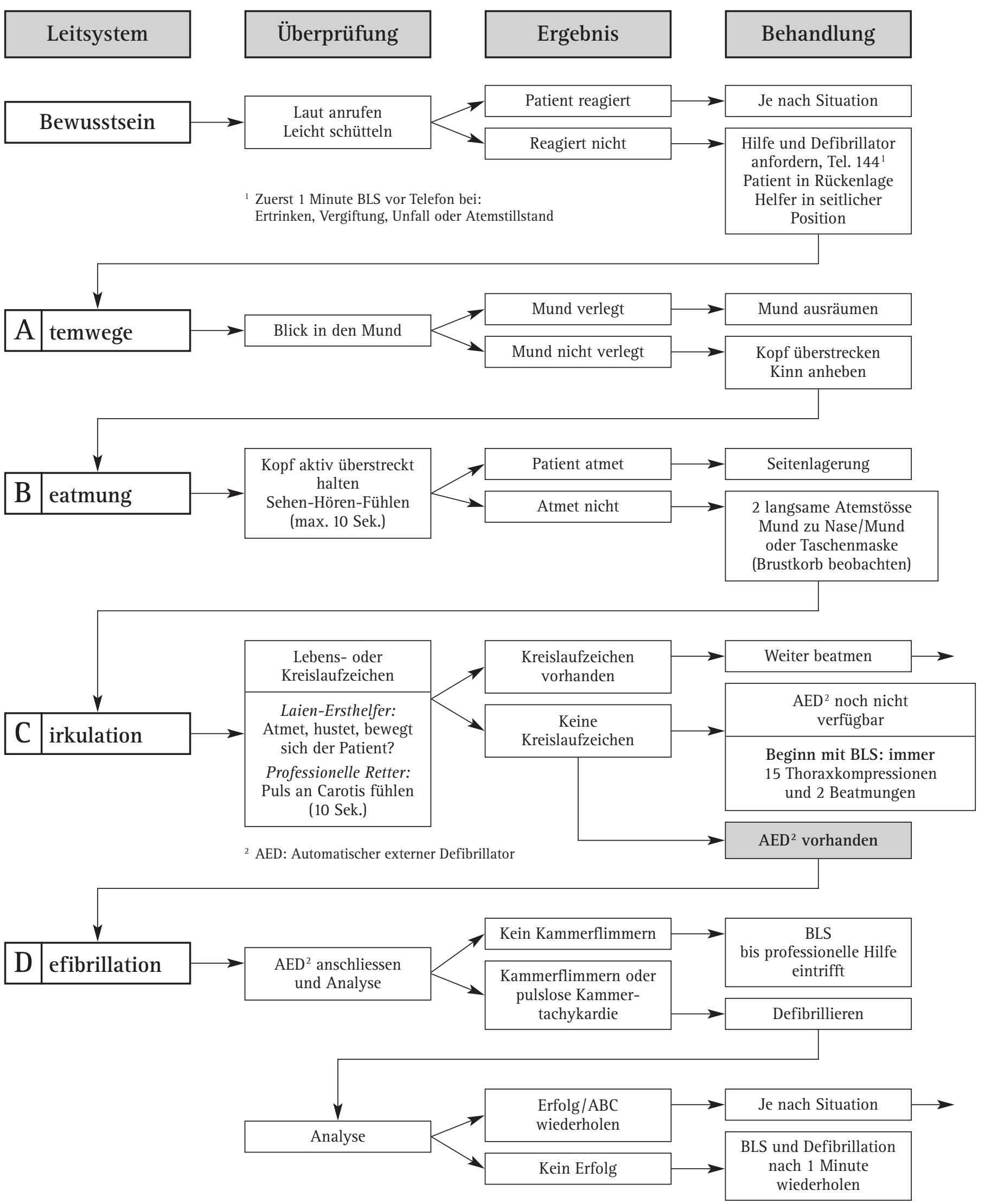


\section{Abbildung 4}

BLS-AED-Vorgehen bei Kreislaufstillstand.

Liegt ein Kreislaufstillstand vor:

Patienten in Rückenlage bringen, Notfallsystem aktivieren, Defibrillator organisieren, mit BLS beginnen.

Wenn Defibrillator vorhanden Rhythmusanalyse über die Defibrillator-Elektroden: falls Schock empfohlen sofortige Defibrillation!

\begin{tabular}{|l|r|c|c|}
\hline Leitsystem & Überprüfung & Ergebnis & Behandlung \\
\hline Bewusstsein & Laut anrufen, leicht schütteln & Keine Reaktion & $\begin{array}{c}\text { Notfallsystem aktivieren } \\
\text { Tel. 144, Defibrillator anfordern }\end{array}$ \\
\hline Atemwege & Blick in den Mund & Verlegt & Mund ausräumen \\
\cline { 3 - 4 } & Sehen, Hören, Fühlen der Atmung & Keine Atmung & $\begin{array}{c}\text { Kopf überstrecken, } \\
\text { Unterkiefer anheben }\end{array}$ \\
\hline${ }^{*}$ Cirkulation & $\begin{array}{r}\text { Laien: Atmet, hustet, bewegt Patient } \\
\text { Profis: Carotispuls (10 Sek.) }\end{array}$ & Keine Kreislaufzeichen & $\begin{array}{c}\text { Beginn mit BLS bis } \\
\text { Defibrillator vorhanden }\end{array}$ \\
\end{tabular}

* Pulskontrolle von Laien-Ersthelfern nicht verlangt.

BLS und automatische externe Defibrillation.

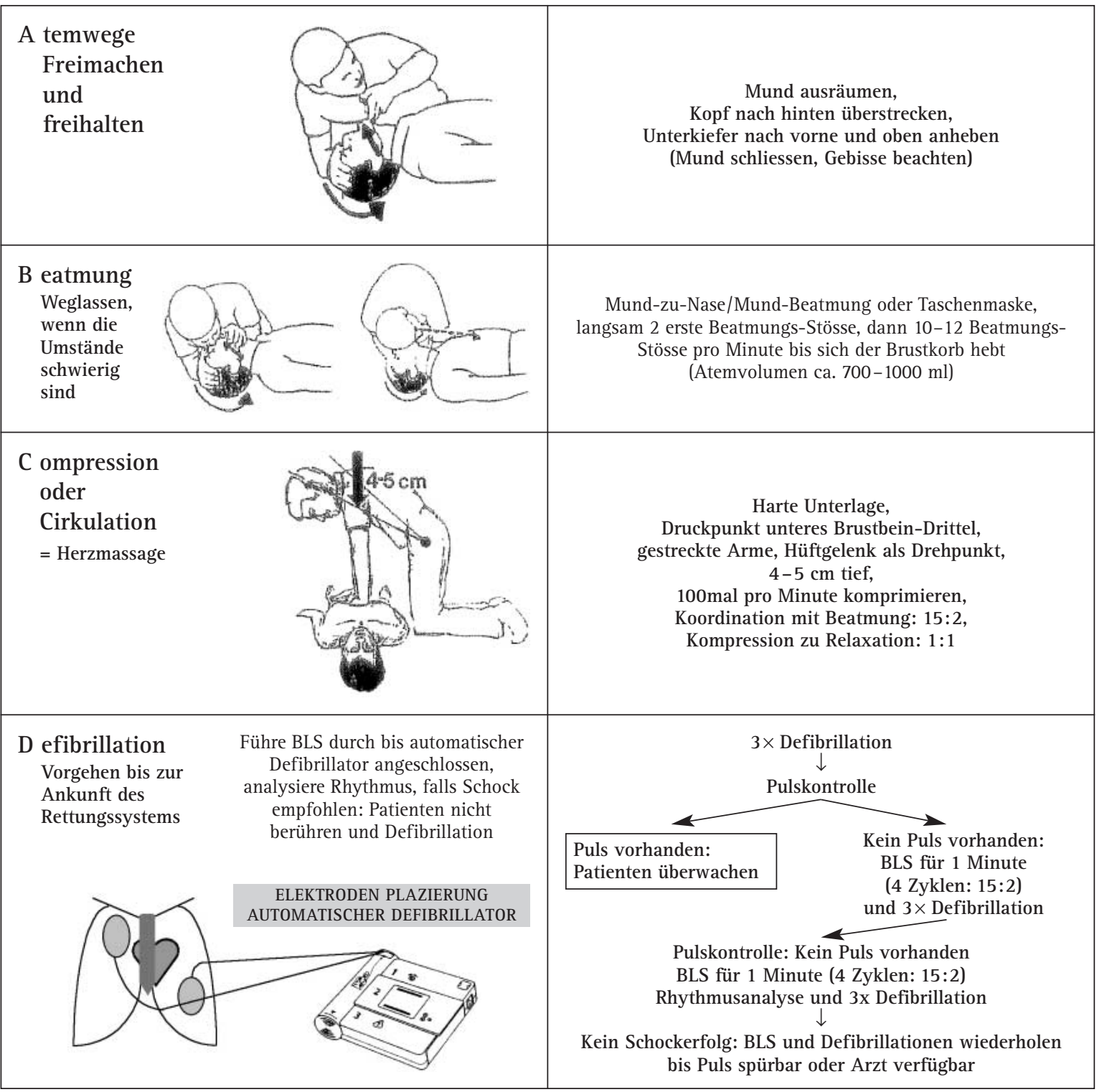




\section{Abbildung 5}

ACLS-CPR-Ergänzung bei Kreislaufstillstand.

\begin{tabular}{|l|l|l|l|l|}
$\begin{array}{l}\text { Infektionsgefahr } \\
\text { (Hepatitis, Herpes, HIV, Tbc) }\end{array}$ & $\begin{array}{l}\text { Prävention durch Einsatz von Latex-Handschuhen, Masken- oder Beutelbeatmung } \\
\text { (Tücher oder ähnliches wenig wirksam) }\end{array}$ \\
\hline Rhythmusanalyse & Durch Monitorfunktion des automatischen Defibrillators & Vorgehen \\
Heimlich-Manöver (Bolus-Aspiration) & $\begin{array}{l}\text { Am Kopf des Pat. knien, } \\
\text { Beutel auf Oberschenkel } \\
\text { ausdrücken, Gesichts- } \\
\text { maske mit anderer Hand } \\
\text { fest auf das Gesicht } \\
\text { drücken, } 0_{2} \text { anschliessen. }\end{array}$
\end{tabular}

Mund-zu-Maske-Beatmung

- Maske fest auf die Gesichtskontur drücken,

- Mit Zeigefinger und Daumen Maske halten,

- Beatmen und die Thoraxexkursionen beobachten.

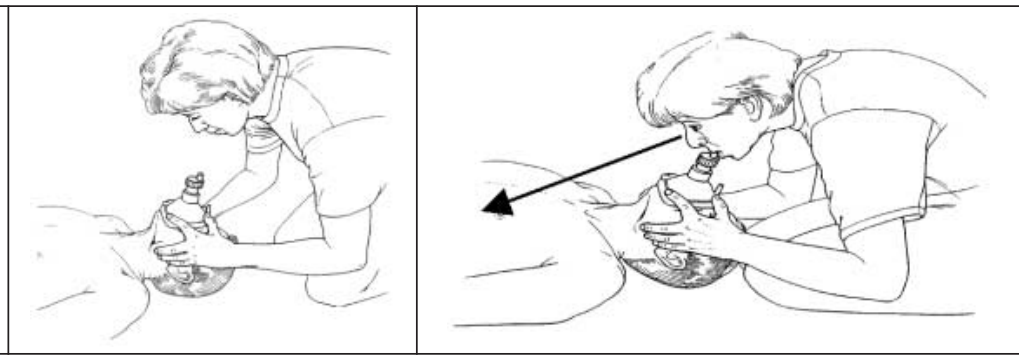

\begin{tabular}{|c|c|}
\hline Venöser Zugang & Kurzer Katheter in Ellenbeuge oder Vorderarm $(0,9 \% \mathrm{NaCl})$ \\
\hline \multirow[b]{2}{*}{$\begin{array}{l}\text { i.v. Medikation immer } \\
\text { mit } 20 \mathrm{ml} 0,9 \% \mathrm{NaCl} \\
\text { flushen }\end{array}$} & $\begin{array}{l}\Uparrow \text { Cardiac output und Blutdruck } \\
\text { Adrenalin: } 1 \mathrm{mg} \text { alle } 3-5 \text { Min, Vasopressin: } 40 \mathrm{U} 1 \times \text { Bolus }\end{array}$ \\
\hline & $\begin{array}{l}\text { Arrhythmietherapie } \\
\text { Amiodaron: } 300 \mathrm{mg} \text { iv, 2. Dosis: } 150 \mathrm{mg} \text { iv (max. } 2.2 \mathrm{gr} / 24 \mathrm{~h} \text { ) } \\
\text { Lidocain: } 1-1.5 \mathrm{mg} / \mathrm{kg} \text { alle } 3-5 \mathrm{Min}(\mathrm{max} .3 \mathrm{mg} / \mathrm{kg} \text { ) } \\
\text { Procainamid: } 30 \mathrm{mg} / \mathrm{kg} \text { bei refraktärer } \mathrm{KT} / \mathrm{KF} \\
\text { Magnesium-Sulfat: } 1-2 \mathrm{gr} \text { bei Torsade de pointes } \\
\text { Atropin: } 1 \mathrm{mg} \text { alle } 3-5 \mathrm{Min} \text { (max. } 3 \mathrm{mg} \text { ) }\end{array}$ \\
\hline
\end{tabular}

\section{Abbildung 6}

Gegenüberstellung GABI - ABCD.

\begin{tabular}{|c|c|c|c|}
\hline \multicolumn{2}{|c|}{ GABI } & \multicolumn{2}{c|}{ ABCD } \\
\hline & & Bewusstsein & \\
\hline G & Gibt der Patient Antwort & Atemwege & A \\
\hline A & Atmet der Patient & Beatmung & B \\
\hline B & Blutet der Patient & Cirkulation & C \\
\hline I & Ist ein Puls spürbar & Defibrillation & D \\
\hline
\end{tabular}

